\title{
Sarcopenia and the elusive fountain of youth
}

\author{
Stephen B Hanauer
}

Nass and colleagues (Nass $R$ et al. [2008] Ann Intern Med 149: 601-611) state that "Aging is an inevitable process across all species" and "Frailty is one of the scourges of elderly persons." Sarcopenia, the age-related decrease in skeletal muscle mass and strength, is a major risk factor for frailty, loss of independence, and physical disability in the elderly. Therapies that prevent or reverse sarcopenia and other hormone-related changes associated with aging have the potential to reduce the morbidity of aging adults and their resultant dependency on families and society.

Ghrelin, a hormone produced in the stomach, small intestine and hypothalamus, increases appetite and exerts growth-hormone-releasing effects. As aging is associated with decreases in growth hormone secretion and circulating concentrations of insulin-like growth factor I (IGF-I), one potential, gut-centric approach to the prevention or treatment of sarcopenia (and frailty) involves the use of a ghrelin mimetic such as MK-677. This orally active growthhormone secretagogue and growth-hormone secretagogue receptor agonist can restore serum levels of IGF-I in aging individuals to those of young adults.

Nass and colleagues investigated the effects of MK-677 on age-associated changes in body composition (decreased lean body mass and increased body fat) in a 2-year, double-blind, crossover study that enrolled healthy men and women aged 60-81 years who received morning doses of $25 \mathrm{mg} \mathrm{MK}-677$ or placebo. The primary outcome measures were changes in fat-free mass and abdominal visceral fat at 12 months. Secondary outcome assessments included growth-hormone secretion profiles and morning IGF-I serum concentrations, as well as other

\section{Therapies \\ that prevent \\ or reverse \\ sarcopenia and other hormone- related \\ changes \\ associated \\ with aging have \\ the potential \\ to reduce the \\ morbidity of \\ aging adults \\ and their \\ resultant \\ dependency \\ on families and \\ society}

SB Hanauer is Editorin-Chief of Nature Clinical Practice Gastroenterology \& Hepatology.

Competing interests

The author declared no competing interests.

www.nature.com/clinicalpractice doi:10.1038/ncpgasthep1320 components of body composition, strength, physical performance and quality of life. The authors confirmed that MK-677 augmented circadian growth-hormone secretion and morning serum IGF-I concentrations to those of healthy young adults, and increased total lean body mass and intracellular water (a biomarker of fatfree mass). Although total body fat and abdominal fat, muscle strength and quality of life did not change, serum levels of LDL cholesterol decreased while cortisol, fasting blood glucose and glycated hemoglobin levels increased. As expected, appetite and body weight increased; however, previously described undesirable consequences of growth-hormone replacement therapy including peripheral edema, myalgias, arthralgias, and new malignancies were not detected in this study population of otherwise healthy 'seniors'.

As pointed out by the authors and in an editorial published in the same issue (Blackman MR [2008] Ann Intern Med 149: 677679), the study population was compromised by the enrollment of a relatively small number of healthy participants (both men and women) without consideration of their pretreatment hormone levels or use of hormone replacement therapy. Furthermore, these patients were followed up for only 12 months. Nevertheless, the study by Nass and colleagues sets the stage for future studies of interventions that aim to modify the natural processes of healthy and pathologic aging. Although an optimal intervention paradigm remains elusive, we can ponder whether Juan Ponce de León, the $15^{\text {th }}$ century Spanish conquistador thought to have searched for the Fountain of Youth in presentday Florida, should perhaps have looked inward, rather than outward. 